\title{
Examination of the Effect of Rehabili-Mouse, a Desktop Rehabilitation Robot for Upper Limb Paresis after Stroke
}

\author{
Rena Wakabayashi ${ }^{*}$, Kimio Saito ${ }^{2}$, Toshiki Matsunaga², Satoaki Chida², Kai Kagami², \\ Takehiro Iwami ${ }^{3}$, Satoru Kizawa4, Yuki Terata ${ }^{5}$, Masumi Ogasawara6, Yoichi Shimada ${ }^{7}$, \\ Naohisa Miyakoshi ${ }^{1}$
}

${ }^{1}$ Department of Orthopedic Surgery, Akita University Graduate School of Medicine, Akita, Japan

${ }^{2}$ Department of Rehabilitation Medicine, Akita University Hospital, Akita, Japan

${ }^{3}$ Department of Mechanical Engineering, Akita University, Akita, Japan

${ }^{4}$ National Institute of Technology, Akita College, Akita, Japan

${ }^{5}$ Faculty of Systems Science and Technology, Akita Prefectural University, Akita, Japan

${ }^{6}$ Oyu Rehabilitation Hot-Spring Hospital, Kazuno, Japan

${ }^{7}$ Akita Prefectural Center on Development and Disability, Akita, Japan

Email: *roughpon15@gmail.com

How to cite this paper: Wakabayashi, R. Saito, K., Matsunaga, T., Chida, S., Kagami, K., Iwami, T., Kizawa, S., Terata, Y., Ogasawara, M., Shimada, Y. and Miyakoshi, N. (2021) Examination of the Effect of Rehabili-Mouse, a Desktop Rehabilitation Robot for Upper Limb Paresis after Stroke. Open Journal of Orthopedics, 11, 371-382. https://doi.org/10.4236/ojo.2021.1112035

Received: November 12, 2021 Accepted: December 11, 2021 Published: December 14, 2021

Copyright $\odot 2021$ by author(s) and Scientific Research Publishing Inc. This work is licensed under the Creative Commons Attribution International License (CC BY 4.0).

http://creativecommons.org/licenses/by/4.0/

\begin{abstract}
Background: Active rehabilitation of the paralyzed limb is necessary for functional recovery from upper limb paralysis after stroke. In particular, the amount of training is very important, and robot rehabilitation is useful. However, most conventional robots are expensive, large, and stationary. We have developed Rehabili-Mouse, a new tabletop rehabilitation robot that is compact and portable. The purpose of this study was to conduct paralyzed upper limb training for a patient after stroke using Rehabili-Mouse and to examine its effect. Case: The patient was a 44-year-old man who had left-sided paresis after a right cerebral infarction, 3 months after onset. The training was carried out between February 2021 and March 2021 at Oyu Rehabilitation Hot-spring Hospital. The training was 20 minutes of Rehabili-Mouse in addition to 40 minutes of usual occupational therapy and performed five times a week for four weeks. Upper limb functions were evaluated before and after the training, and two questionnaires of patient satisfaction with the device and the training were administered after the completion of the training. Upper limb function improved. The patient's satisfaction with the device was poor, but his satisfaction with the training was good. Discussion: Training for the paralyzed upper limb after stroke using Rehabili-Mouse improved upper limb function and satisfied the trained patient. We plan to increase the number of cases and conduct further studies.
\end{abstract}




\section{Keywords}

Robotic Rehabilitation, Hemiplegia, Upper Limb Function

\section{Introduction}

Stroke is a disease that causes severe functional declines in physical and cognitive functions, and about $70 \%$ of survivors have temporary upper limb dysfunction [1]. Of these, only about $15 \%$ are reported to have useful upper limb function, [2] and upper limb paralysis leads to a decrease in quality of life (QOL) [3]. Therefore, active rehabilitation of the paralyzed limb is necessary for functional recovery from upper limb paralysis caused by stroke. In particular, the amount of training is very important [4] [5], and robotic rehabilitation is considered to be useful in this regard [6].

Previous reports have indicated that assist-as-needed type training tasks encourage upper limb participation and increase the number of training repetitions [7]. In fact, in stroke patients, it has been shown that the higher the intensity of upper limb robot treatment, the better the upper limb function [8]. Furthermore, the combination of upper limb robot treatment and conventional rehabilitation treatment is reportedly more effective than either treatment alone [9], and robot rehabilitation for paralyzed limbs is being put to practical use. It is also expected to be applied in clinics and for home use as an alternative or superior method to the conventional approach [10].

Rehabilitation robots can perform training and evaluation simultaneously. Their advantages as training devices are that they are good at repetitive movements and adjusting tasks according to the patient's ability. Their advantages as measurement devices are that they are easy to program, the results are objective and accurate, and the difficulty of the task can be easily adjusted based on external disturbances [11]. It has also been reported that they can provide a standardized environment and reduce the burden on therapists [12]. Furthermore, conventional rehabilitation methods often involve repetitive daily tasks, which may cause patients to lose interest in the treatment [13]. Rehabilitation using games not only improves physical functions, but also has psychological effects, leading to motivation to use the paralyzed limb and achieve goals in real life [14], and robot rehabilitation is considered useful in this respect as well.

However, most conventional robots are expensive, large, and stationary, which are barriers to the introduction of robotic rehabilitation. Therefore, we developed a new type of tabletop rehabilitation robot, "Rehabili-Mouse", which is compact and portable, and it can be easily used on a desktop to rehabilitate upper limb paresis [15].

The purpose of this study was to examine the therapeutic effects of paralyzed upper limb training using the Rehabili-Mouse in a subacute and chronic stroke patient with upper limb paralysis. 


\section{Case}

\subsection{Inclusion and Exclusion Criteria}

The inclusion criteria were: 1) subacute or chronic upper limb paresis patient with stroke; and 2) the person could give written, informed consent after reviewing a consent explanation document.

The exclusion criteria were: 1) skin disorders of the forearm and hand; 2) history of epilepsy; 3 ) uncontrolled disease; 4) lack of understanding and motivation for treatment; 5) pregnancy; 6) severe joint contracture or deformity; and 7) under 18 years of age.

\subsection{Ethical Considerations}

Prior to subject recruitment, a review was performed by the Ethics Committee of the Certified Clinical Research Review Board, Akita University, and the Ministry of Health, Labour and Welfare, and approval was received (Acceptance No. A2020-01, jRCTs022200014). All subjects gave written, informed consent prior to screening procedures and recruitment.

\subsection{Period and Place}

This study was conducted at Oyu Rehabilitation Hot-spring Hospital between February 2021 and March 2021.

\subsection{Participant}

The participant was a 44-year-old, right-handed man. He had left-sided paresis after a right cerebral infarction, 3 months after onset (Table 1). He was the first patient to start a study and meet the conditions at our hospital.

\subsection{Equipment and Systems}

The Rehabili-Mouse (Figure 1) comprises a desktop rehabilitation robot, a monitor, an infrared sensor camera for position measurement (HTC Taiwan: Base Station + VIVE Tracker), and a laptop PC for operation. The robot body is very compact, disk-shaped, approximately $300 \mathrm{~mm}$ in diameter, $150 \mathrm{~mm}$ in height, and $7.0 \mathrm{~kg}$ in weight, with a handle on the top to move it. The handle is connected internally to a force sensor that activates the internal motor in response to forces exerted on it by the subject. The robot has a servomotor and four omnidirectional drive wheels that enable it to move in any direction on a plane (Figure 2). The strength of the assist by the robot can be adjusted, and training can be performed according to the degree of paralysis and recovery. The robot is powered using a portable battery. Participants sit in chairs and operate the robot on the table while viewing the monitor located $500 \mathrm{~mm}$ in front of them (Figure 3). The positional information is detected by the infrared sensor of the Base Station system and incorporated into the software. As a safety device, the movement of the Rehabili-Mouse can be set to any range in the initial setting. Therefore, the movement is limited and the robot stops moving when it is about to go 
Table 1. Characteristics of the patient.

\begin{tabular}{cc}
\hline & case \\
\hline Sex & Male \\
Age & 44 \\
Dominant hand & Right \\
Paralyzed side & Left \\
Primary disease & right cerebral infarction \\
Disease period & 3 months \\
\hline
\end{tabular}

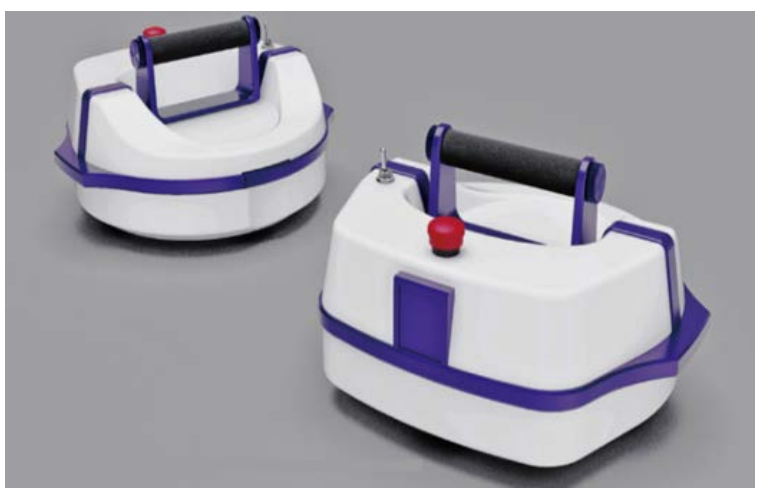

Figure 1. Rehabili-Mouse, our upper limb rehabilitation robot: disk-shaped, approximately $300 \mathrm{~mm}$ in diameter, $150 \mathrm{~mm}$ in height, and $7.0 \mathrm{~kg}$ in weight.

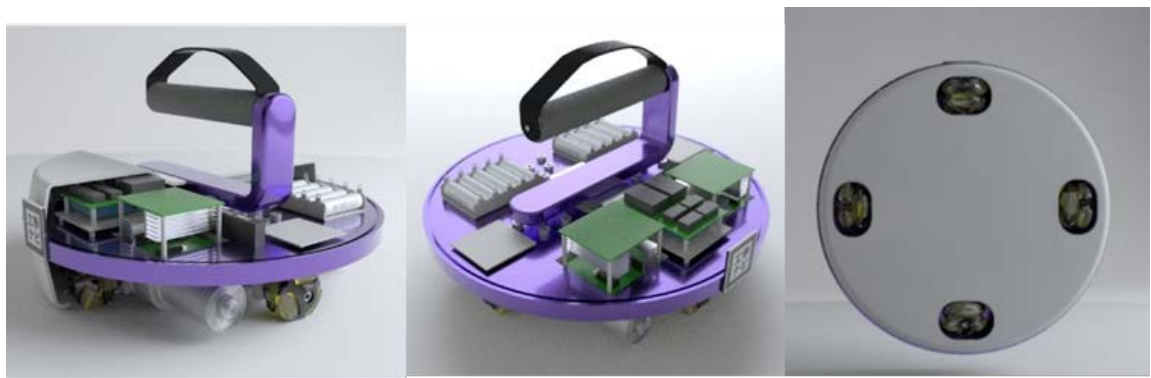

Figure 2. Internal structure of the Rehabili-Mouse with the cover removed, and the bottom: It has a servomotor and four omnidirectional drive wheels, and it is driven by applying force to the handle on the top connected to a force sensor. The robot is powered by a portable battery.

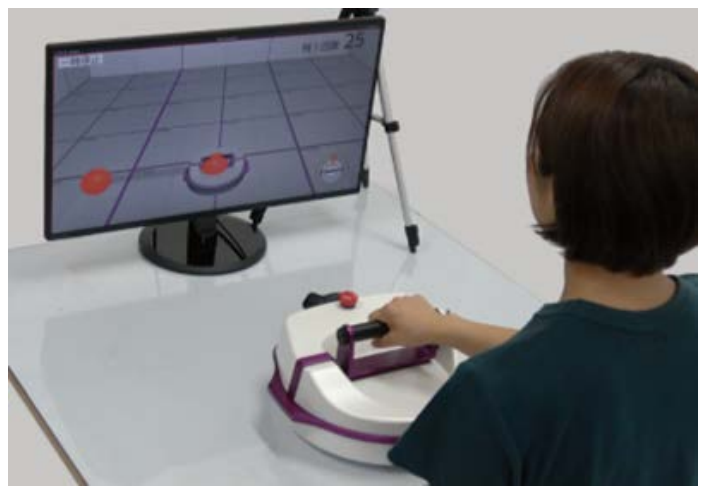

Figure 3. Example of the training set-up: Participants sit in chairs and operate the robot on the table while viewing the monitor located $500 \mathrm{~mm}$ ahead. 
out of range. In addition, a load sensor is attached to the handle, and the robot stops moving when the hand is released from the handle. These safety functions can prevent the Rehabili-Mouse from falling off the desktop or malfunctioning during training. No dangerous situations or safety concerns were encountered in our previous study [15]. The training software was originally developed with fun and motivation in mind, and it includes five basic motion training exercises (forward-backward reaching, five-way sector reaching, eight-way reaching, and clockwise and counterclockwise circular orbit training) and game training exercises (air hockey, a frustrating bar game, kart racing game, and whack-a-mole) (Figure 4). The motion trajectories are recorded, and the game scores are displayed.

\subsection{Procedure}

In addition to 40 minutes of usual occupational therapy, training with the Rehabili-Mouse was performed for 20 minutes under the supervision of an occupational therapist (OT), referring to previous studies [16] [17]. The training was conducted five times a week for four weeks.

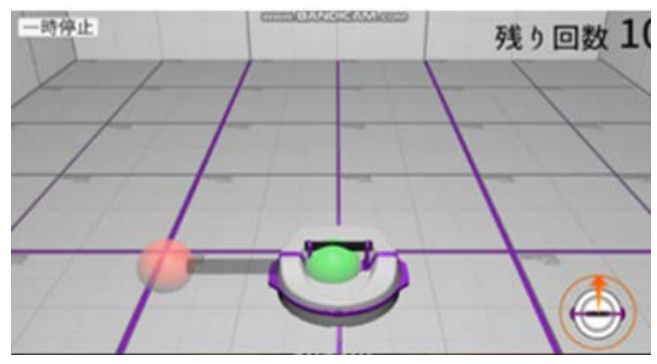

(a)

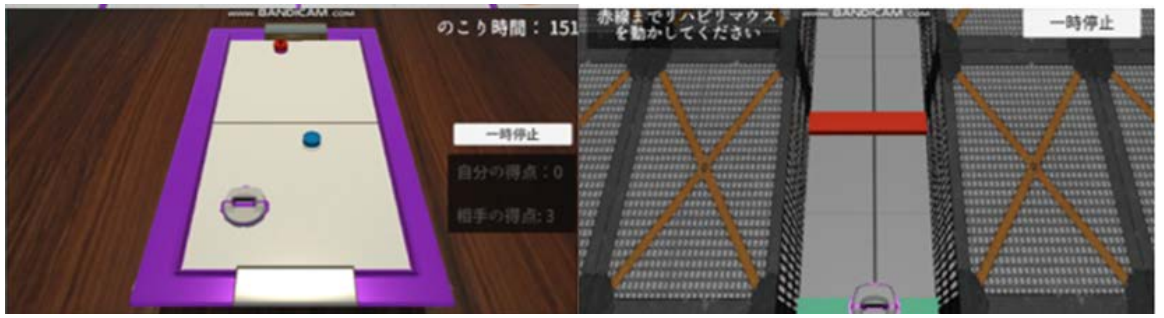

(b)

(c)

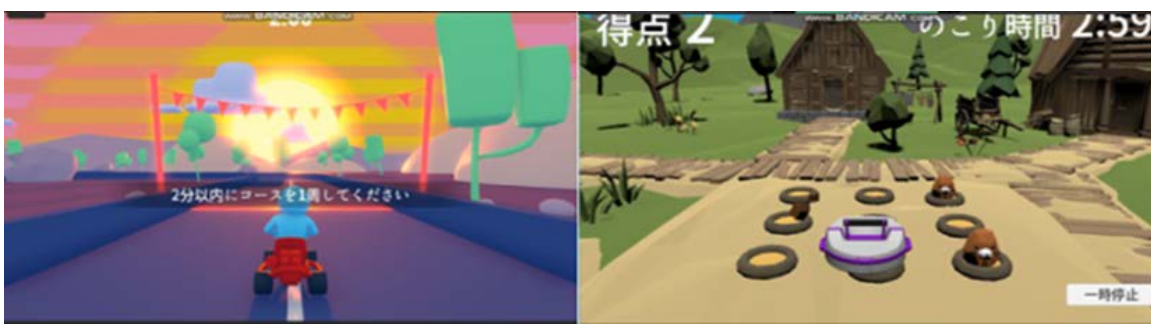

(d)

(e)

Figure 4. The training software: Original software that includes five basic motion training (e.g., (a) eight-way reaching) and game training exercises ((b) air hockey, (c) a frustrating bar game, (d) kart racing game, and (e) whack-a-mole) was developed. 


\subsection{Assessment Parameters}

Before and after training, Brunnstorm stage (Br. stage), Fugl-Meyer Assessment (FMA), Modified Ashworth Scale (MAS), Action Research Arm Test (ARAT), Functional Independence Measure (FIM), and Motor Activity Log (MAL) were evaluated. In addition, two types of questionnaires were administered after the four-week training period was completed. The first one was the System Usability Scale (SUS) [18] about the equipment, and the second one was our original questionnaire combining several questions [19] about the training. Both questionnaires use a five-point self-evaluation scale ranging from 1 , strongly disagree, to 5 , strongly agree.

\section{Results}

Upper limb function improved as follows: Br. stage from V/IV to V/V; FMA total score from 119 to 125 ; MAS from 1/1/1/1 to 0/1/1/0 for shoulder/elbow/hand/ finger joints; ARAT from 48 to 57; FIM from 73 to 126; and MAL from 2.8/3.4 to 4.9/4.1 for Amount of Use (AOU)/Quality of Movement (QOM) (Table 2). The SUS score was 55, Grade D (Poor) (Table 3), and the results of the original questionnaire for training satisfaction were 3 for "6. Did you feel discomfort during your experience with the system?" and "8. Do you want to continue using this system?", and 4 - 5 for Others (Table 4).

\section{Discussion}

In the present study, upper limb functional training was conducted for a hemiplegic patient after a stroke using our device, and upper limb function and patient satisfaction were evaluated. The results of the upper limb evaluation (FMA, MAS, ARAT, FIM, and MAL) ranged from unchanged to improved. Patient satisfaction was high for the training, but low for SUS related to the system.

Robot rehabilitation is evidence level A in the American Heart Association/ American Stroke Association (AHA/ASA) guidelines [20] and according to a review by Langhorne et al. [21] and is strongly recommended for rehabilitating

Table 2. Results of upper limb function before and after the training.

\begin{tabular}{cccc}
\hline & Before Training & After Training & Change \\
\hline Br. Stage & V/VI & V/V & \\
FMA/126 & 119 & 125 & 6 \\
MAS & $1 / 1 / 1 / 1$ & $0 / 1 / 1 / 0$ & $1 / 0 / 0 / 1$ \\
ARAT/57 & 38 & 57 & 9 \\
FIM & 73 & 126 & 53 \\
AML (AOU/QOM) & $2.8 / 3.4$ & $4.9 / 4.1$ & $2.1 / 0.7$ \\
\hline
\end{tabular}

Br. stage, Brunnstorm stage; FMA, Fugl-Meyer Assessment; MAS, Modified Ashworth Scale; ARAT, Action Research Arm Test; FIM, Functional Independence Measure; MAL, Motor Activity Log. 
Table 3. Results of the original questionnaire for training after the completion of the 4-week training period.

\begin{tabular}{lc}
\hline \multicolumn{1}{c}{ Question } & $\begin{array}{c}\text { 1 Not at All - } \\
\text { 5 Very Much }\end{array}$ \\
\hline 1) Did you have the motivation to get good scores? & 5 \\
2) Did you enjoy your experience with the system? & 5 \\
3) Were you successful using the system? & 4 \\
4) Were you able to control the system? & 5 \\
5) Is the information provided by the system clear? & 3 \\
6) Did you feel discomfort during your experience with the system? & 3 \\
7) Do you think that this system will be helpful for your rehabilitation? & 4 \\
8) Do you want to continue using this system? & 3 \\
9) Other Comments & More games \\
& would be nice. \\
\hline
\end{tabular}

Table 4. Results of the system usability scale (SUS) for patient satisfaction with the device after the completion of the 4 -week training period.

\begin{tabular}{|c|c|}
\hline Question & $\begin{array}{l}1 \text { strongly disagree } \\
\text { - } 5 \text { strongly agree }\end{array}$ \\
\hline 1) I think that I would like to use this system frequently. & 2 \\
\hline 2) I found the system unnecessarily complex. & 4 \\
\hline 3) I thought the system was easy to use. & 4 \\
\hline $\begin{array}{l}\text { 4) I think that I would need the support of a technical person to be } \\
\text { able to use this system. }\end{array}$ & 1 \\
\hline 5) I found the various functions in this system were well integrated. & 1 \\
\hline 6) I thought there was too much inconsistency in this system. & 5 \\
\hline $\begin{array}{l}\text { 7) I would imagine that most people would learn to use this system } \\
\text { very quickly. }\end{array}$ & 4 \\
\hline 8) I found the system very cumbersome to use. & 2 \\
\hline 9) I felt very confident using the system. & 2 \\
\hline $\begin{array}{l}\text { 10) I needed to learn a lot of things before I could get going with } \\
\text { this system. }\end{array}$ & 2 \\
\hline SUS Score & 55 \\
\hline
\end{tabular}

upper limb dysfunction in patients with cerebrovascular disease. Its usefulness was also reported in the 2018 Cochrane review [22] and meta-analysis, and it is effective in improving the function of the paralyzed hand when performed in addition to the usual rehabilitation by therapists [6]. The Rehabili-Mouse is smaller, lighter, and less expensive than the other upper limb rehabilitation robots reported previously. In addition, it does not need to be operated by technical specialists, and can be operated by the rehabilitation staff of the hospital. Thus, we are considering the possibility of using it in clinics and at home in the future.

In the present case, upper limb function improved, as follows: MAS in the shoulder joint and hand; FMA, especially in the hand joint, hand, and sensation; FIM in all items; MAL in both AOU and QOM; and ARAT in Pinch. That is, not only function, but also use of the paralyzed limb in activities of daily living (ADL) 
was improved. In recent years, the gap between upper limb function and ADL after training with robotic rehabilitation has been pointed out [23]. This suggests that the function improved by robotic therapy is unlikely to affect the actual activities in daily life. In the present study, not only upper limb function, but also upper limb ADL evaluation was improved. It is thought that the combined training of normal rehabilitation by a therapist and robotic rehabilitation increased the frequency of use of the paralyzed hand, reduced spasticity, and improved function, and thus improved the ADL of the paralyzed limb. This is consistent with previous reports [24] [25], which demonstrated that the number of repetitions is important for changes in neural structure and motor function after brain injury, i.e., brain plasticity. However, improvement in the natural course of disease cannot be ruled out, and comparison with a control group is needed. In addition, there is a report that $\mathrm{ADL}$ was improved by combining robot therapy with constraint-induced movement (CI) therapy [26], and it is thought that the habit of using paralyzed limbs in daily life, that is, the "Transfer Package", is important. We will consider devising combined occupational therapy with robot therapy and developing a system that can be used with augmented reality technology.

It has been suggested that training using robots is effective not only in improving physical function, but also psychological aspects, such as improving patient independence, which increases motivation for using the paralyzed limb in daily life and achieving goals. Conducting rehabilitation through playing games makes the repetitive exercises more engaging and motivating [14]. On the questionnaire regarding patient satisfaction in the present study, aside from one negative response about feeling some discomfort (Table 3 shaded cell), the patient provided three or more positive answers about using the system. During the training, comments from the participant included, "It was interesting," "I was happy to win the game or to improve my score," "I felt lighter in my upper limbs," and "I realized that I was not good at upper limb abduction." Taub et al. [27] reported that, even in patients with chronic stroke, intensive training for a certain period of time can improve the function of the upper limbs. Miyakoshi et al. [28] suggested that their original Virtual Reality (VR) upper limb function recovery training device could give clear goals through game-like tasks, and that motor learning effects could be obtained by practicing these tasks. The present device is also game-like, and the visually clear goals and feedback of training results will likely facilitate motivation, concentration, and continuity. Furthermore, its force sensor and the motor-assisted movements allow patients to adjust the difficulty level according to the degree of paralysis and to perform the movements they are not good at with support, so that they can unconsciously concentrate on such training. The results of SUS for the system were low. The reason why the result was low for questions such as " 5 . I found the various functions in this system were well integrated." and "6. I thought there was too much inconsistency in this system." is thought to be that many problems, such as screen 
malfunctions, occurred due to a system error during training. The results for question " 4 . I think that I would need the support of a technical person to be able to use this system." and "9. I felt very confident using the system." were also low, but in this study, the equipment was operated by rehabilitation staff. Although it is not necessary to have a device expert, it is considered reasonable to have a staff member who understands how to operate the equipment accompany the patient during training. The evaluation for the question regarding the versatility of the device, "7. I would imagine that most people would learn to use this system very quickly." was good. Currently, we have been improving the system errors, which are considered to be one of the factors directly related to patient satisfaction with our device, by correcting them each time they occur. The therapist also commented that "in addition to the muscle-strengthening effect, the frustrating bar game is good training for coordination," and "since the patient knew all the contents of the game in the final week, it would be better to add levels to the game, in addition to the game types." In order to achieve effective functional improvement, it is necessary to set up detailed training according to the individual patient's background and function [23]. Therefore, we plan to enhance the software of our device and improve the game further.

\section{Limitations}

This was a case report, and usual rehabilitation by therapists was also performed. To examine the effect of robot therapy more convincing, it is necessary to increase the number of cases and compare it with a self-training group (control group) in the future. Then, further research development can be expected based on this preliminary result. Since the data was only recorded before and after training, by recording the data 6 weeks and more after the completion of the training and examining whether the training effect is sustained, it could be more progressive study. In addition, because system errors occurred, a survey will be conducted after correcting the problems so that the full potential of the device can be demonstrated. Furthermore, follow-up after the evaluation was not performed, so subsequent follow-up studies should be considered.

\section{Conclusion}

Paralyzed upper limb training was conducted using the Rehabili-Mouse in a patient with paresis of an upper limb after stroke. Upper limb function improved, the patient's satisfaction with the training was high, and the procedure was safe. We plan to increase the number of cases and conduct further studies.

\section{Acknowledgements}

The authors would like to express their sincere gratitude to everyone involved in the development of the device, all those who supported us, and the patient who participated in this study. This work did not receive any grants from funding agencies in the public, commercial, or not-for-profit sectors. 


\section{Conflicts of Interest}

There are no conflicts of interest to declare.

\section{References}

[1] Nakayama, H., Jorgensen, H.S., Raaschou, H.O. and Olsen, T.S. (1994) Recovery of Upper Extremity Function in Stroke Patients: The Copenhagen Stroke Study. Archives of Physical Medicine and Rehabilitation, 75, 394-398.

https://doi.org/10.1016/0003-9993(94)90161-9

[2] Sunderland, A., Tinson, D.J., Bradley, E.L., Fletcher, D., Langton-Hewer, R. and Wade, D.T. (1992) Enhanced Physical Therapy Improves Recovery of Arm Function after Stroke. A Randomised Controlled Trial. Journal of Neurology, Neurosurgery and Psychiatry, 55, 530-535. https://doi.org/10.1136/jnnp.55.7.530

[3] Nichols-Larsen, D.S., Clark, P.C., Zeringue, A., Greenspan, A. and Blanton, S. (2005) Factors Influencing Stroke Survivors' Quality of Life during Subacute Recovery. Stroke, 36, 1480-1484. https://doi.org/10.1161/01.STR.0000170706.13595.4f

[4] Kwakkel, G., Wagenaar, R.C., Koelman, T.W., Lankhorst, G.J. and Koetsier, J.C. (1997) Effects of Intensity of Rehabilitation after Stroke. A Research Synthesis. Stroke, 28, 1550-1556. https://doi.org/10.1161/01.STR.28.8.1550

[5] Han, C., Wang, Q., Meng, P.-P. and Qi, M.-Z. (2012) Effects of Intensity of Arm Training on Hemiplegic Upper Extremity Motor Recovery in Stroke Patients: A Randomized Controlled Trial. Clinical Rehabilitation, 27, 75-81. https://doi.org/10.1177/0269215512447223

[6] Norouzi-Gheidari, N., Archambault, P.S. and Fung, J. (2012) Effects of Robot-Assisted Therapy on Stroke Rehabilitation in Upper Limbs: Systematic Review and Meta-Analysis of the Literature. JRRD, 49, 479-496.

https://doi.org/10.1682/JRRD.2010.10.0210

[7] Page, S.J., Schmid, A. and Harris, J.E. (2012) Optimizing Terminology for Stroke Motor Rehabilitation: Recommendations from the American Congress of Rehabilitation Medicine Stroke Movement Interventions Subcommittee. Archives of Physical Medicine and Rehabilitation, 93, 1395-1399. https://doi.org/10.1016/j.apmr.2012.03.005

[8] Burgar, C.G., Lum, P.S., Scremin, A.M., Garber, S.L., Vander Loos, H.F., Kenney, D. and Shor, P. (2011) Robot-Assisted Upper-Limb Therapy in Acute Rehabilitation Setting Following Stroke: Department of Veterans Affairs Multisite Clinical Trial. Journal of Rehabilitation Research \& Development, 48, 445-458. https://doi.org/10.1682/JRRD.2010.04.0062

[9] Zhang, C., Li-Tsang, C.W.P.P. and Au, R.K. (2017) Robotic Approaches for the Rehabilitation of Upper Limb Recovery after Stroke: A Systematic Review and Meta-Analysis. International Journal of Rehabilitation Research, 40, 19-28. https://doi.org/10.1097/MRR.0000000000000204

[10] Gassert, R. and Dietz, V. (2018) Rehabilitation Robots for the Treatment of Sensorimotor Deficits: A Neurophysiological Perspective. JNER, 15, 46.

https://doi.org/10.1186/s12984-018-0383-x

[11] Volpe, B.T., Huerta, P.T., Zipse, J.L., Rykman, A., Edwards, D., Dipietro, L., Hogan, N. and Krebs, H.I. (2009) Robotic Devices as Therapeutic and Diagnostic Tools for Stroke Recovery. Archives of Neurology, 66, 1086-1090. https://doi.org/10.1001/archneurol.2009.182

[12] Jakob, I., Kollreider, A., Germanotta, M., Benetti, F., Cruciani, A., Padua, L. and 
Aprile, I. (2018) Robotic and Sensor Technology for Upper Limb Rehabilitation. $P M R, 10$, S189-S197. https://doi.org/10.1016/j.pmrj.2018.07.011

[13] Burdea, G. (2003) Virtual Rehabilitation-Benefits and Challenges. Methods of Information in Medicine, 42, 519-523. https://doi.org/10.1055/s-0038-1634378

[14] Chen, Y., Abel, K.T., Janecek, J.T., Chen, Y., Zheng, K. and Cramer, S.C. (2019) Home-Based Technologies for Stroke Rehabilitation. A Systematic Review. International Journal of Medical Informatics, 123, 11-22. https://doi.org/10.1016/j.ijmedinf.2018.12.001

[15] Mizutani, T., Matsunaga, T., Saito, K., Iwami, T., Kizawa, S., Anbo, T. and Simada, Y. (2017) Development of Tabletop Robot for Rehabilitation of Upper Limb Paralysis and Application to Assess Upper Limb Function. Akita Journal of Medicine, 44, 111-116.

[16] Hesse, S., Schulte-Tigges, G., Konrad, M., Bardeleben, A. and Werner, C. (2003) Robot-Assisted Arm Trainer for the Passive and Active Practice of Bilateral Forearm and Wrist Movements in Hemiparetic Subjects. Archives of Physical Medicine and Rehabilitation, 84, 915-920. https://doi.org/10.1016/S0003-9993(02)04954-7

[17] Hesse, S., Werner, C., Pohl, M., Rueckriem, S., Mehrholz, J. and Lingnau, M.L. (2005) Computerized Arm Training Improves the Motor Control of the Severely Affected Arm after Stroke: A Single-Blinded Randomized Trial in Two Centers. Stroke, 36, 1960-1966. https://doi.org/10.1161/01.STR.0000177865.37334.ce

[18] Brooke, J. (1996) SUS-A Quick and Dirty Usability Scale. In: Jordan, P.W., Thomas, B., Weerdmeester, B.A. and McClelland, I.L., Eds., Usability Evaluation in Industry, Taylor \& Francis, London, 189-194.

[19] Gil-Gómez, J.A., Manzano-Hernández, P., Albiol-Pérez, S., Aula-Valero, C., Gil-Gómez, H. and Lozano-Quilis, J.A. (2017) USEQ: A Short Questionnaire for Satisfaction Evaluation of Virtual Rehabilitation Systems. Sensors, 17, 1589. https://doi.org/10.3390/s17071589

[20] Winstein, C.J., Stein, J., Arena, R., Bates, B., Cherney, L.R., Cramer, S.C., Deruyter, F., Eng, J.J., Fisher, B., Harvey, R.L., Lang, C.E., MacKay-Lyons, M., Ottenbacher, K.J., Pugh, S., Reeves, M.J., Richards, L.G., Stiers, W. and Zorowitz, R.D. (2016) Guidelines for Adult Stroke Rehabilitation and Recovery: A Guideline for Healthcare Professionals from the American Heart Association/American Stroke Association. Stroke, 47, e97-e169. https://doi.org/10.1161/STR.0000000000000098

[21] Langhorne, P., Bernhardt, J. and Kwakkel, G. (2011) Stroke Rehabilitation. The Lancet, 377, 1693-1702. https://doi.org/10.1016/S0140-6736(11)60325-5

[22] Mehrholz, J., Pohl, M., Platz, T., Kugler, J. and Elsner, B. (2015) Electromechanical and Robot-Assisted Arm Training for Improving Activities of Daily Living, Arm Function, and Arm Muscle Strength after Stroke. Cochrane Database of Systematic Reviews, No. 11, CD006876. https://doi.org/10.1002/14651858.CD006876.pub4

[23] Takahashi, K., Domen, K., Sakamoto, T., Toshima, M., Otaka, Y., Seto, M., Irie, K., Haga, B., Takebayashi, T. and Hachisuka, K. (2016) Efficacy of Upper Extremity Robotic Therapy in Subacute Post-Stroke Hemiplegia: An Exploratory Randomized Trial. Stroke, 47, 1385-1388. https://doi.org/10.1161/STROKEAHA.115.012520

[24] Nudo, R.J., Wise, B.M., SiFuentes, F. and Milliken, G.W. (1996) Neural Substrates for the Effects of Rehabilitative Training on Motor Recovery after Ischemic Infarct. Science, 272, 1791-1794. https://doi.org/10.1126/science.272.5269.1791

[25] Krakauer, J.W., Carmichael, S.T., Corbett, D. and Witten-berg, G.F. (2012) Getting Neurorehabilitation Right: What Can Be Learned from Animal Models? Neurore- 
habilitation and Neural Repair, 26, 923-931. https://doi.org/10.1177/1545968312440745

[26] Takebayashi, T., Amano, S., Hanada, K., Umeji, A., Takahashi, K., Koyama, T. and Domen, K. (2014) Therapeutic Synergism in the Treatment of Post-Stroke Arm Paresis Utilizing Botulinum Toxin, Robotic Therapy, and Constraint-Induced Movement Therapy. PM R, 6, 1054-1058. https://doi.org/10.1016/j.pmrj.2014.04.014

[27] Taub, E., Miller, N.E., Novack, T.A., Cook, E.W., Fleming, W.C., Nepomuceno, C.S., Connell, J.S. and Crago, J.E. (1993) Technique to Improve Chronic Motor Deficit after Stroke. Archives of Physical Medicine and Rehabilitation, 74, 347-354.

[28] Miyakoshi, K., Domen, K., Koyama, T., Furusho, J. and Koyanagi, K. (2006) The Effect of Robot-Aided Training on Motor Recovery Following Stroke. The Japanese Journal of Rehabilitation Medicine, 43, 347-352.

https://doi.org/10.2490/jirm1963.43.347 